\title{
Is liaison psychiatry effective in primary care? ${ }^{\dagger}$ COMMENTARY ON... COCHRANE CORNER
}

\author{
Alex J. Mitchell
}

\section{SUMMARY}

'Collaborative care' (involving a case manager) and 'primary care liaison' or 'consultation liaison' (with no case manager) are models of liaison psychiatry in primary care. Here, I briefly consider the evidence for collaborative care, discuss Gillies et al's Cochrane review on consultation liaison, and suggest avenues for future study and development of liaison psychiatry in primary care.

\section{DECLARATION OF INTEREST}

None

There is increasing awareness of comorbidity in physical and mental illness and the need for equal consideration of both areas (parity of esteem). Despite this, many people with mental health complications of physical illness do not receive prompt treatment. Liaison psychiatry (known as consultation-liaison psychiatry in some countries) is traditionally considered an effective way of improving mental healthcare for people in hospital, but increasing the value of liaison psychiatry is being tested in primary care (Ruddy 2005; Sved Williams 2006). In the primary care context it has been termed 'collaborative care' when a case manager (also called a care manager or case worker) is involved and 'primary care liaison' or 'consultation liaison' when there is no case manager. In collaborative care, primary care staff work together with one or more mental health specialists (psychiatrist, mental health nurse, psychologist or suitably trained social worker), often supplemented by a team or case manager, to care for the patient. The attraction of collaborative care and primary care liaison is that primary care is usually the first port of call for patients with mental health problems and most mental illness is managed solely in primary care (World Health Organization 2001). Primary care has no shortage of patients with medically unexplained symptoms, chronic physical illness and unrecognised psychiatric comorbidity who would benefit from specialist input. Primary care liaison has the potential to improve the ability of primary care providers to recognise and treat mental disorders (Younès 2008) as well as improving use of scarce mental health resources (Mitchell 2002; Sved Williams 2006). However, there has been uncertainty whether this model is truly effective in routine clinical practice and whether it offers benefits over usual care and/or other models of healthcare.

\section{Evidence base for collaborative care}

The evidence supporting collaborative care involving a case manager has been extensively debated, particularly as studies have tended to be short term and resource intensive. The collaborative care model emphasises the use of a case manager to facilitate outcome monitoring, self-management, treatment adherence and, when needed, specialist consultation (Roy-Byrne 2013). Collaborative care's key criteria are nothing new, namely a multiprofessional approach to patient care, structured management, scheduled patient follow-ups and enhanced communication between professionals. However, allocation of a specific case manager helps clarify areas of responsibility, enhances communication and promotes optimal

\section{BOX 1 Liaison psychiatry in primary care}

Collaborative care: the patient's mental healthcare is managed by a case manager, enhancing the care from the patient's primary care provider working with one or more attached mental health specialists

Primary care liaison: the primary care provider maintains the central role in delivering mental healthcare, with an attached mental health specialist providing consultative support but without a case manager.

Case manager (care manager, case worker): this is a healthcare professional responsible for coordination of mental health by promoting and monitoring optimal care pathways and tracking clinical outcomes and coordinating clinical care and communication. In short, they help ensure timely good-quality care.

\section{ROUND THE} CORNER
Alex J. Mitchell is a consultant psychiatrist in the Department of Psycho-Oncology, Leicestershire Partnership NHS Trust and the Department of Cancer and Molecular Medicine, University of Leicester. Correspondence Dr Alex J. Mitchell, Department of PsychoOncology, Hadley House, Leicester General Hospital, Leicester LE5 4PW, UK. Email ajm80@le.ac.uk

${ }^{\dagger}$ See p. 74, this issue. 
care pathways. Collaborative care clinicians usually deliver evidence-based psychotherapies or evidence-based pharmacotherapy (or both).

Initial evidence was promising. An early Cochrane review that included 79 randomised controlled trials (RCTs) and 24308 participants showed that collaborative care was more effective than usual care for both depression and anxiety immediately after treatment and up to 2 years later (Archer 2012; Reilly 2013). A meta-analysis of 32 RCTs of collaborative care published between 2004 and 2009 found evidence of effectiveness in improving symptoms, adherence to treatment, response to treatment and satisfaction with care for patients diagnosed with depression (Thota 2012). A systematic review and meta-regression focusing on what made collaborative care effective included 74 RCTs and 21345 participants suffering from mood disorders (Coventry 2014). It reported that collaborative care that included psychological interventions predicted improvement in depression. Systematic identification of patients (i.e. screening tools $)(P=0.004)$ and the presence of a chronic physical condition $(P=0.02)$ were linked with better adherence with antidepressant medication. One criticism is that most studies of collaborative care have been short term and based in the USA, but a new review of 15 RCTs in European countries appears to offer some reassurance of improvement in depression outcomes at 12 months and over (Sighinolfi 2014).

Thus, the evidence appears to suggest that collaborative care (defined as use of case manager alongside mental health professionals attached to primary care) is effective, but is a case manager really an essential component?

\section{Gillies et al's Cochrane review}

The review featured in this month's Cochrane Corner (Gillies 2015) examined studies published up to March 2014 on the effectiveness of primary care liaison $v$. other types of mental healthcare. The authors attempted to separate primary care liaison (which they refer to as 'consultation liaison') from collaborative care on the basis of whether there was a primary care case manager: in primary care liaison no case manager was involved. The authors found 12 trials involving 2605 patients across 905 primary care providers. Eight trials included people with depression, and the rest involved patients with mixed mood disorders. None focused on severe mental illness. This omission is not unique: the evidence for collaborative care in severe mental illness is also scant.
B0X 2 Bias

Bias is defined as any systematic error in an epidemiological study that results in an incorrect estimate of the association between exposure and risk of disease. In clinical trials, bias may distort the outcomes of the intervention compared with the control. Although over 50 varieties of bias have been identified in clinical research, the common types seen in psychiatric research include reporting bias, performance bias (mentioned here), selection bias, recall bias, confirmation bias and attrition bias.

Looking at the methodological quality of the studies, the sample size of all subgroups was small. Eight trials were rated at high risk of performance bias (Box 2) because participants were likely to have known whether or not they were allocated to the intervention group and because most outcomes were self-reported. Bias due to attrition was rated high in eight trials and reporting bias was rated high in six. The types of interaction that took place in the interventions included: for primary care providers assistance with assessment interviews and with referrals to specialist mental healthcare; for patients, psychoeducation, counselling, structured advice, treatment monitoring and provision of self-help. Most interactions were face to face, but some trials included only contact by telephone (of 1-12 sessions).

The authors' analysis provided some evidence that primary care liaison improved patients' mental health at least up to 3 months following the start of treatment, but there was no evidence of core effectiveness beyond 3 months, and in any case the longest follow-up was 12 months. Regarding secondary outcomes such as patient satisfaction and adherence to treatment, primary care liaison appeared to improve patient satisfaction and adherence for up to 12 months. Regarding primary care clinicians' behaviour, there was also an improvement in adequate treatment between 3 to 12 months. By the authors' own rating, the quality and reliability of these findings were low for all outcomes (apart from adherence, which was of moderate quality). It is important to remember that given the issue with allocation concealment (above), it is quite likely that ratings from patients (and assessors) favoured the new intervention rather than usual care, especially when one arm was more intensive.

Eleven trials compared primary care liaison with a standard care, randomising either patients or GPs or practices, but only one was conducted 
in the UK (Drummond 1990). One trial with 354 patients and 67 primary care providers randomised patients to a case manager (in effect comparing primary care liaison with collaborative care) (Hedrick 2003). In this head-to-head study, collaborative care produced greater improvement than primary care liaison in depressive symptoms from baseline to 3 months, but at 9 months there was no significant difference, calling into question the added benefit of the case manager if other systems are in place.

\section{Conclusions}

Overall Gillies et al's Cochrane review provides modest evidence that primary care liaison (consultation liaison) improves some mental health outcomes up to 3 months, as well as improving satisfaction and adherence up to 12 months, in people with mental disorders, particularly those who are depressed. However, there is a need for caution given low sample sizes, lack of allocation concealment, low study quality and short follow-up durations. With only one study, there is little to suggest that collaborative care is fundamentally superior to primary care liaison . What is uncertain is whether either collaborative care or primary care liaison would out-perform alternative active models of mental healthcare in which equivalent resources of collaborative care were invested. In fact, given that two key elements of effective care include psychological interventions and systematic identification (or screening), other models of care incorporating these elements could be compared with primary care liaison and/or collaborative care. For example, how would the Improving Access to Psychological Treatments (IAPT) programme compare with collaborative care? Which would be more cost-effective? Two reviews of collaborative care found only modest evidence for cost-effectiveness (van SteenbergenWeijenburg 2010; Grochtdreis 2015). Comparison of incremental cost-effectiveness ratios (ICERs) for improvements in quality of life between IAPT and collaborative care would be of interest (compared with Mukuria et al (2013) for example). Finally, a major question remains over rates of uptake of collaborative care, which may be as low as $11 \%$ even from a pool of suitable patients (Kendrick 2013). Even if collaborative care and primary care liaison are effective, the model used in clinical practice must be acceptable to clinicians and patients alike.

\section{References}

Archer J, Bower P, Gilbody S, et al (2012) Collaborative care for depression and anxiety problems. Cochrane Database of Systematic Reviews, 10: CD006525.

Coventry PA, Hudson JL, Kontopantelis E, et al (2014) Characteristics of effective collaborative care for treatment of depression: a systematic review and meta-regression of 74 randomised controlled trials. PLoS One, 9(9): e108114.

Drummond DC, Thom B, Brown C, et al (1990) Specialist versus general practitioner treatment of problem drinkers. Lancet, 336: 915-8.

Gillies D, Buykx P, Parker AG, et al (2015) Consultation liaison in primary care for people with mental disorders. Cochrane Database of Systematic Reviews, 9: CD007193.

Grochtdreis T, Brettschneider C, Wegener A, et al (2015) Cost-effectiveness of collaborative care for the treatment of depressive disorders in primary care: a systematic review. PLoS One, 10(5): e0123078.

Hedrick SC, Chaney EF, Felker B, et al (2003) Effectiveness of collaborative care depression treatment in Veterans' Affairs primary care. Journal of General Internal Medicine, 18: 9-16.

Kendrick TA (2013) Clinical effectiveness of collaborative care for depression in UK primary care (CADET): cluster randomised controlled trial. BMJ, 347: f4913.

Mitchell G, Del Mar C, Francis D (2002) Does primary medical practitioner involvement with a specialist team improve patient outcomes? A systematic review. British Journal of General Practice, 52: 934-9.

Mukuria C, Brazier J, Barkham M, et al (2013) Cost-effectiveness of an Improving Access to Psychological Therapies service. British Journal of Psychiatry, 202: 220-7

Reilly S, Planner C, Gask L, et al (2103) Collaborative care approaches for people with severe mental illness. Cochrane Database of Systematic Reviews, 11: CD009531.

Roy-Byrne P (2013) Collaborative care at the crossroads. British Journal of Psychiatry, 203: 86-7.

Ruddy R, House A (2005) Meta-review of high-quality systematic reviews of interventions in key areas of liaison psychiatry. British Journal of Psychiatry, 187: 109-20.

Sighinolfi C, Nespeca C, Menchetti M, et al (2014) Collaborative care for depression in European countries: a systematic review and metaanalysis. Journal of Psychosomatic Research, 77: 247-63.

Sved Williams A, Dodding J, Wilson I, et al (2006) Consultation-liaison to general practitioners coming of age: the South Australian psychiatrists' experience. Australasian Psychiatry, 14: 206-11.

Thota AB, Sipe TA, Byard GJ, et al (2012) Collaborative care to improve the management of depressive disorders: a community guide systematic review and meta-analysis. American Journal of Preventive Medicine, 42: 525-38.

van Steenbergen-Weijenburg KM, van der Feltz-Cornelis CM, Horn EK, et al (2010) Cost-effectiveness of collaborative care for the treatment of major depressive disorder in primary care: a systematic review. BMC Health Services Research, 10: 19.

World Health Organization (2001) The World Health Report 2001 Mental Health: New Understanding, New Hope. WHO

Younès N, Passerieux C, Hardy-Bayle MC, et al (2008) Long term GP opinions and involvement after a consultation-liaison intervention for mental health problems. BMC Family Practice, 9: 41. 